\title{
DISCRETE TIME S-I-S MODELS WITH SIMPLE AND COMPLEX POPULATION DYNAMICS
}

BU-1539 -M
October, 2000

\section{Carlos Castillo-Chavez and \\ Abdul-Aziz Yakabu}

Keywords: none provided.

\begin{abstract}
The model for this study is an extension of a nonlinear discrete-time susceptibleinfected-susceptible (S-I-S) epidemic model of Barrera et al. and Velazquez that includes populations exhibiting geometric, bounded or complex dynamics. Thresholds for disease persistence are computed and used to illustrate the analysis of the asymptotic global behavior of solutions. Extensions and results that include SIS models capable of supporting multiple attractors are discussed
\end{abstract}




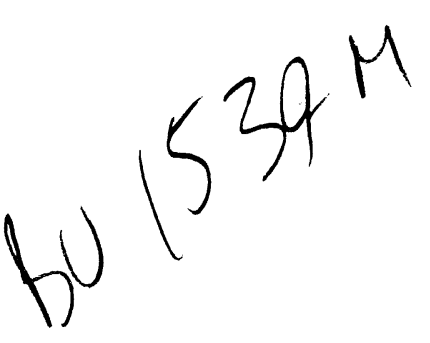

\title{
DISCRETE-TIME S-I-S MODELS WITH SIMPLE AND COMPLEX POPULATION DYNAMICS
}

\author{
Carlos Castillo-Chavez \\ Biometrics Department \\ Cornell University \\ Ithaca, New York 14853 \\ and \\ Abdul-Aziz Yakubu \\ Department of Mathematics \\ Howard University \\ Washington, DC 20059
}

October 18, 2000

\begin{abstract}
The model for this study is an extension of a nonlinear discretetime susceptible-infected-susceptible $(S-I-S)$ epidemic model of Barrera et $a l$. and Velazquez that includes populations exhibiting geometric, bounded or complex dynamics. Thresholds for disease persistence are computed and used to illustrate the analysis of the asymptotic global behavior of solutions. Extensions and results that include SIS models capable of supporting multiple attractors are discussed.
\end{abstract}




\section{Introduction}

Simple nonlinear discrete-time population models are capable of generating complex (chaotic) dynamics $[4,6,9,18,21,22]$. A simple nonlinear discretetime susceptible-infected-susceptible $(S-I-S)$ epidemic model that assumes that the population is either asymptotically bounded, experiences geometric growth or is capable of generating complex dynamics is presented.

Theoretical studies of $(S-I-S)$ epidemic models have been carried out in deterministic (discrete and continuous-time) and stochastic Markov chains for a long time (see Allen [1, 2]; Anderson et al. [3]; Bailey [5]; Castillo et al. [7 - 10]; Cooke and Yorke [11]; Hassell et al. [18] and Nåsell [23], to name a few). Barrera et al. [6]; Velazquez [27]; and Castillo-Chavez and Yakubu $[9,10]$ have developed models for the study of disease dynamics in populations with discrete generations and potentially complex (chaotic) disease-free dynamics. The models in this article overlap somewhat with $(S-I-S)$ epidemic models found in the literature (see $[6,9,10,16,27])$.

\section{Simple Discrete-time Epidemic Models}

Nonlinear discrete-time epidemic models are built under the assumption that the dynamics of the total population size in generation $n$, denoted by $T(n)$, are governed by an equation of the form $T(n+1)=f(T(n))+\gamma T(n)$, where

$0 \leq \gamma<1$ denotes the constant probability of surviving per generation and $f$ models the typically nonlinear birth or recruitment process. If $f$ is not typical, that is, if $f$ is a constant or a linear function of the total population then the population dynamics are rather simple. Specifically, if new recruits 
arrive at the constant per-capita rate $\mu$ or at the total constant rate $\Lambda$ per generation then $f(T(n))=\mu T(n)$ or $f(T(n))=\Lambda$. An epidemic process is built on "top" of this demographic model that, by construction, has known disease-independent, and simple population dynamics for $T(n)$. Epidemics on simple demographic settings are the norm in the study of continuoustime epidemic models where the total population is typically assumed to be constant, asymptotically constant, or capable of supporting exponential growth. This framework expands the range of complexity of the underlying population dynamics (see the next section for examples).

At generation $n, S(n)$ denotes the population of susceptibles; $I(n)$ denotes the population of infecteds, assumed infectious; $T(n) \equiv S(n)+I(n)$ denotes the total population size; and, $T_{\infty} \equiv \lim _{n \rightarrow \infty} T(n)$ denotes the demographic steady state for the total population whenever it exists. Individuals survive from one generation to the next with constant probability $\gamma$ while infected, individuals recover with constant probability $1-\sigma$ per generation. Susceptible individuals (given that they had contacts with infectives) become infected with probability $1-G$ or, equivalently, remain susceptible with probability $G$ per generation. $G:[0, \infty) \rightarrow[0,1]$ is a monotone concave function with $G(0)=1 ; G^{\prime}(x)<0$ and $G^{\prime \prime}(x) \geq 0$ for all $x \in[0, \infty)$.

The population dynamics are fixed since it is assumed that the disease is not fatal. All recruits are susceptible, time is measured in generations, and recovery from the disease does not give permanent or temporary immunity. The modeling process implicitly assumes a sequential process: At the end of each generation, a fraction $(1-\gamma)$ of each class is removed (death); surviving susceptibles become infected with probability $(1-G)$; and, independently, 
surviving infectives recuver with probability $(1-\sigma)$. It is important to note that this is a deterministic model and that probabilistic lingo is used mainly to facilitate understanding.

Our assumptions lead to the following discrete-time $S-I-S$ epidemic model:

$$
\left.\begin{array}{l}
S(n+1)=f(T(n))+\gamma S(n) G\left(\frac{\alpha I(n)}{T(n)}\right)+\gamma I(n)(1-\sigma), \\
I(n+1)=\gamma\left(1-G\left(\frac{\alpha I(n)}{T(n)}\right)\right) S(n)+\gamma \sigma I(n),
\end{array}\right\}
$$

where $0<\gamma, \sigma<1$, and $T(n)>0$. The constant $\alpha$ weighs the role of prevalence $\left(\frac{I(n)}{T(n)}\right)$ on disease transmission. If $\alpha$ is large, then it is easier to become infected (see Figure 1). System (1), is an extension of the discrete time $(S-I-S)$ epidemic models of Barrera et al. and Velazquez [6, 27].

Since $T(n+1) \equiv S(n+1)+I(n+1)$, then

$$
T(n+1)=f(T(n))+\gamma T(n)
$$

that is, Equation (2) models the dynamics (demography) of the total population.

\section{Simple Population Dynamics and SIS Epi- demics}

If $f(T(n))=\mu T(n)$ then Equation (2), the demographic equation, reduces to the linear difference equation

$$
T(n+1)=(\mu+\gamma) T(n)
$$

and, consequently,

$$
T(n)=(\mu+\gamma)^{n} T(0) .
$$


The demographic basic repruductive number,

$$
\Re_{d}=\frac{\mu}{1-\gamma}
$$

is a dimensionless quantity. It gives the average number of descendants produced by a (typically) small pioneer population $(T(0))$ over its life-time. Hence, if $\Re_{d}>1$, the population invades at a geometric rate and if $\Re_{d}<1$ the population dies geometrically. The epidemic process constructed then lives in a population experiencing geometric dynamics.

If we let

$$
x(n)=\frac{S(n)}{T(n)} \text { and } y(n)=\frac{I(n)}{T(n)},
$$

then System (1) with $f(T(n))=\mu T(n)$ reduces to the one-dimensional autonomous "system" for $y(n)$ :

$$
y(n+1)=\frac{\gamma}{\gamma+\mu}(1-y(n))(1-G(\alpha y(n)))+\frac{\gamma \sigma}{\gamma+\mu} y(n)
$$

Since $x(n)+y(n)=1$ for all $n$ then all solutions live on the invariant line $\{(x, y) \in[0, \infty) \times[0, \infty) \mid x+y=1\}$.

$\Re_{0}$, the number of secondary infections generated by an infected (assumed infectious) individual over his or her lifetime in a population of susceptibles, determines whether or not the disease can invade. From Equation (6), we find that

$$
\Re_{0}=\frac{-\alpha \gamma G^{\prime}(0)}{(1-\gamma)\left(\Re_{d}-1\right)+(1-\gamma \sigma)},
$$

after noticing that near the equilibrium point $\left(x_{\infty}, y_{\infty}\right) \equiv(1,0)$,

$$
y(n+1) \approx \frac{\gamma}{\gamma+\mu}\left(-\alpha G^{\prime}(0)+\frac{\gamma \sigma}{\gamma+\mu}\right) y(n) .
$$


Whenever $\Re_{d}=1$ (no demographic impact), the basic reproductive number, $\Re_{0}$, reduces to $\Re_{0}=\frac{-\kappa \gamma G^{\prime}(0)}{1-\gamma \sigma}$, where $\frac{1}{1-\gamma \sigma}$ denotes the average death-adjusted length of the infectious period in generations; $\gamma$ is the proportion of surviving susceptibles who can be invaded by the disease; and, $-\alpha G^{\prime}(0)$ is the maximum rate of infection per infective $[9,10]$. Since $\frac{1}{(1-\gamma)\left(\Re_{d}-1\right)+(1-\gamma \sigma)}$ gives the demographic death-adjusted infectious period measured in generations then $\Re_{0}$ decreases with population growth $\left(\Re_{d}>1\right)$ and increases with population decay $\left(0<\Re_{d}<1\right)$, that is, whenever $\Re_{d} \neq 1$ demography has an impact.

Result 1. (a) Suppose that $\Re_{d}>1$, that is, assume $T$ is increasing at the geometric rate, $(\mu+\gamma)$. Then

(i) $\Re_{0} \leq 1$, implies that the proportion $\frac{I}{T}$ of infectives in the total population tends to 0 as $n \rightarrow \infty$. Hence, $\left(\frac{S}{T}, \frac{I}{T}\right)$ tends to the disease-free equilibrium $(1,0)$, while $S$ is increasing at the same geometric rate as $T$.

(ii) $\Re_{0}>1$, implies that the proportion $\frac{I}{T}$ of infectives in the total population tends to a positive number, $\frac{\bar{I}}{T}$ as $n \rightarrow \infty$. Hence, $\left(\frac{S}{T}, \frac{1}{T}\right)$ tends to an endemic equilibrium while $I, S$ and $T$ are increasing at the same geometric rate.

(b) Suppose that $\Re_{d}<1$, that is, assume $T$ is decreasing at the geometric rate, $(\mu+\gamma)$. Then

(i) $\Re_{0} \leq 1$, implies that the proportion $\frac{I}{T}$ of infectives in the total population tends to 0 as $n \rightarrow \infty$. Hence, $\left(\frac{S}{T}, \frac{I}{T}\right)$ tends to the disease-free equilibrium $(1,0)$ while $S$ is decreasing to zero al the same geometric rate as $T$.

(ii) $\Re_{0}>1$, implies that the proportion $\frac{I}{T}$ of infectives in the total population tends to a positive number $\frac{\bar{I}}{T}$ as $n \rightarrow \infty$. Hence, $\left(\frac{S}{T}, \frac{I}{T}\right)$ tends to an endemic equilibrium while $I, S$ and $T$ are decreasing at the same geometric 
rate.

Proof: Define the repruduction function for the infected individuals of System (6), $h:[0,1] \rightarrow[0,1]$ by $h(y)=\frac{\gamma}{\gamma+\mu}(1-y)(1-G(\alpha y))+\frac{\gamma \sigma}{\gamma+\mu} y$, where $h(0)=0$ and $0 \leq y \leq 1$. The set of iterates of $h$ is equivalent to the set of density sequence generated by the second equation in System (6). Differentiation with respect to $y$ gives

$$
\begin{aligned}
& h^{\prime}(y)=\frac{\gamma}{\gamma+\mu}\left(-1+G(\alpha y)-\alpha(1-y) G^{\prime}(\alpha y)+\sigma\right) \\
& h^{\prime \prime}(y)=\frac{\gamma}{\gamma+\mu}\left(2 \alpha G^{\prime}(\alpha y)-\alpha^{2}(1-y) G^{\prime \prime}(\alpha y)\right) .
\end{aligned}
$$

$\Re_{0} \leq 1$ implies that $h^{\prime}(0)=\frac{\gamma}{\gamma+\mu}\left(-\alpha G^{\prime}(0)+\sigma\right) \leq 1$. Therefore, the fixed point $\{0\}$ is locally stable under $h$-iteration. Since $G^{\prime}<0$ and $G^{\prime \prime} \geq 0$ we have that, $h^{\prime \prime}(y)<0$ for $y \in[0,1]$. The monotonicity condition on $h^{\prime}$ and the fact that $h^{\prime}(0) \leq 1$ imply that $h^{\prime}(y)<1$ or $h(y)<y$ for $y \in(0,1]$. Hence, $\{y(n)\}_{n \geq 0}$, a strictly decreasing sequence bounded below by zero, converges to the only fixed point of $h$ in the interval $[0,1],\{0\}$. If $\Re_{0} \leq 1$, we have proved that $\frac{I}{T} \rightarrow 0$ as $n \rightarrow \infty$, while the proportion $\frac{S}{T} \rightarrow 1$ as $n \rightarrow \infty$.

$\Re_{0}>1$ implies that $h^{\prime}(0)=\frac{\gamma}{\gamma+\mu}\left(-\alpha G^{\prime}(0)+\sigma\right)>1$ and, therefore, the fixed point $\{0\}$ is locally unstable under $h$-iteration. Let $\bar{y}$ denote the smallest positive fixed point of $h$ in $[0,1]$, and note that $h(1)=\frac{\gamma \sigma}{\gamma+\mu}<1$. The Intermediate Value Theorem guarantees the existence of the positive fixed point $\bar{y} \in(0,1)$ satisfying $h(\bar{y})=\bar{y}$ and $h(y)>y$ for $y \in(0, \bar{y})$ and, consequently, $h^{\prime}(\bar{y}) \leq 1$. Since $h^{\prime \prime}(y)<0$ implies that $h^{\prime}(y)<h^{\prime}(\bar{y}) \leq 1$ for $y \in(\bar{y}, 1)$, then $\int_{\bar{y}}^{y} h^{\prime}(u) d u<\int_{\bar{y}}^{y} d u$ and, we have $h(y)<y$ for $y>\bar{y}$. Hence, $h$ has a unique positive fixed point $\bar{y} \in(0,1)$. Furthermore, $h(y)>y$ for $y \in(0, \bar{y})$ and $h(y)<y$ for $y \in(\bar{y}, 1]$.

To establish the global stability of $\bar{y}$, we first prove the nonexistence of 
non-trivial two-cycles for $h$. Nute that $1+h^{\prime}(y)=1+\frac{\gamma}{\gamma+\mu}(-1+G(\alpha y)-$ $\left.\alpha(1-y) G^{\prime}(\alpha y)+\sigma\right) \geq 1-\frac{\gamma}{\gamma+\mu}+\frac{\gamma \sigma}{\gamma+\mu}>0$. Hence, $1+h^{\prime}(y) \neq 0$ for $y \in[0$, 1], that is, $h$ has no non-trivial 2-cycles. Suppose $h$ has a non-trivial 2-cycle $\{p, q\}$ where $p, q \in[0,1]$, then $h(p)=q$ and $h(q)=p$ where $p \neq q$. The Mean Value Theorem guarantees the existence of a point $\xi$ between $p$ and $q$ such that $h^{\prime}(\xi)=\frac{h(p)-h(q)}{p-q}=-1$, and $1+h^{\prime}(\xi)=0$, a contradiction. Hence, $h$ has no non-trivial 2-cycles in $[0,1]$. Sharkovskii's Theorem and $1+h^{\prime}(y) \neq 0$ imply the nonexistence of any $m-$ cycles for $m>1$. From a result of Cull [12], the nonexistence of non-trivial 2-cycles for $h$ implies global stability of the positive fixed point $\bar{y}$. Hence, $\Re_{0}>1$, implies that $\frac{I}{T} \rightarrow \frac{\bar{I}}{T} \in(0,1)$ as $n \rightarrow \infty$, and $\frac{S}{T} \rightarrow \frac{\bar{S}}{T} \in(0,1)$ as $n \rightarrow \infty$.

A second simple example is provided with $f(T(n))=\Lambda$. The demographic equation, reduces to the linear difference equation

$$
T(n+1)=\Lambda+\gamma T(n)
$$

and, hence

$$
T(n)=\left(T^{\prime}(0)-\frac{\Lambda}{1-\gamma}\right) \gamma^{n}+\frac{\Lambda}{1-\gamma} .
$$

Since $0<\gamma<1$, the total population is asymptotically constant, that is, $T_{\infty}=\frac{\Lambda}{1-\gamma}$, that is, the demographic effects "eventually" disappear.

To study disease epidemics on (7), we assume that the total population $T(n) \equiv S(n)+I(n)$ has reached the positive steady state $T_{\infty}$. If we redefine $S(n)=T_{\infty}-I(n)$ then from System (1) we obtain the following associated one-dimensional dynamically equivalent autonomous "system" for $I(n)[25$, 
28] :

$$
\left.\begin{array}{l}
S(n+1)=f\left(T_{\infty}\right)+c \gamma\left(T_{\infty}-I\right) G\left(\frac{\alpha I(n)}{T_{\infty}}\right)+\gamma I(n)(1-\sigma) \\
I(n+1)=\gamma\left(1-G\left(\frac{\alpha I(n)}{T_{\infty}}\right)\right)\left(T_{\infty}-I\right)+\gamma \sigma I(n) .
\end{array}\right\}
$$

Its basic reproductive number is

$$
\Re_{0}=\frac{-\alpha \gamma G^{\prime}(0)}{1-\gamma \sigma}
$$

and a simple application of earlier results gives the following the result $[9$, 10]:

Result 2: Suppose $f(T(n))=\Lambda$ in system (9) then

(a) If $\Re_{0} \leq 1$, then all solutions $(S(n), I(n))$ approach the globally asymptotically stable disease free equilibrium, $\left(\frac{\Lambda}{1-\gamma}, 0\right)$, as $n \rightarrow \infty$.

(b) If $\Re_{0}>1$, then all solutions $(S(n), I(n))$ approach a unique positive and globally asymptotically stable endemic equilibrium, $(\bar{S}, \bar{I}) \in(0, \infty) \times$ $(0, \infty)$, as $n \rightarrow \infty$.

The proof of Result (2) is the same as that of Theorem (1) in [9].

Consider the following particular version of Model (1):

$$
\left.\begin{array}{l}
S(n+1)=f\left(I^{\prime}(n)\right)+e^{-d} S(n) e^{-\frac{\alpha I(n)}{T(n)}}+e^{-d} I(n)\left(1-e^{-\beta}\right), \\
I(n+1)=e^{-d}\left(1-e^{-\frac{\alpha I(n)}{T(n)}}\right) S(n)+e^{-d} e^{-\beta} I(n),
\end{array}\right\}
$$

where $\alpha, \beta$ and $d$ are positive constants. Here, we have taken $e^{-d}=\gamma$, $e^{-\frac{\alpha I(n)}{T(n)}}=G\left(\frac{\alpha I(n)}{T(n)}\right)$ and $e^{\beta}=\sigma . f(T(n))=\mu T(n)$ implies that $T(n+1)=$ $\left(e^{-d}+\mu\right) T(n)$ and $\Re_{d}=\frac{\mu}{1-e^{-d}}$. Using proportions reduces System (10) to the following system of equations (see, $[6,9,10]$ ):

$$
\left.\begin{array}{l}
x(n+1)=\frac{n}{\epsilon^{-d}+\mu}+\frac{e^{-d}}{\epsilon^{-d}+\mu} x(n) e^{-\alpha y(n)}+\frac{e^{-d}\left(1-e^{-\beta}\right)}{\epsilon^{-d}+\mu} y(n), \\
y(n+1)=\frac{e^{-d}}{\epsilon^{-d}+\mu} x(n)\left(1-c^{-\alpha y(n)}\right)+\frac{e^{-d} e^{-\beta}}{e^{-d}+\mu} y(n) .
\end{array}\right\}
$$


The basic reproductive number,

$$
\Re_{0}=\frac{\alpha^{2}}{1+\mu c^{d}-e^{-\beta}},
$$

determines the asymptutic behaviur of System (11) [see Result 1].

We fix the constants $\beta=0.1, d=\ln 2$ and $\mu=0.1$ while we vary the transmission coefficient $\alpha$. As we increase $\alpha$, the basic reproductive number, $\Re_{0}$, increases from values less than 1 to numbers bigger than 1 . A transcritical bifurcation occurs. The demographic basic reproductive number remains fixed at $\Re_{d}=\frac{\mu}{1-e^{-d}}=0.25<1$. The system with proportions changes from a globally stable disease-free equilibrium to a globally stable endemic equilibrium while the total population is decreasing to zero at a geometric rate (see Figure 1). In particular, at $\alpha=0.6, \Re_{0}=1.22>1$ and the system with proportions has a globally stable endemic equilibrium at $(0.5598,0.4402)$. 


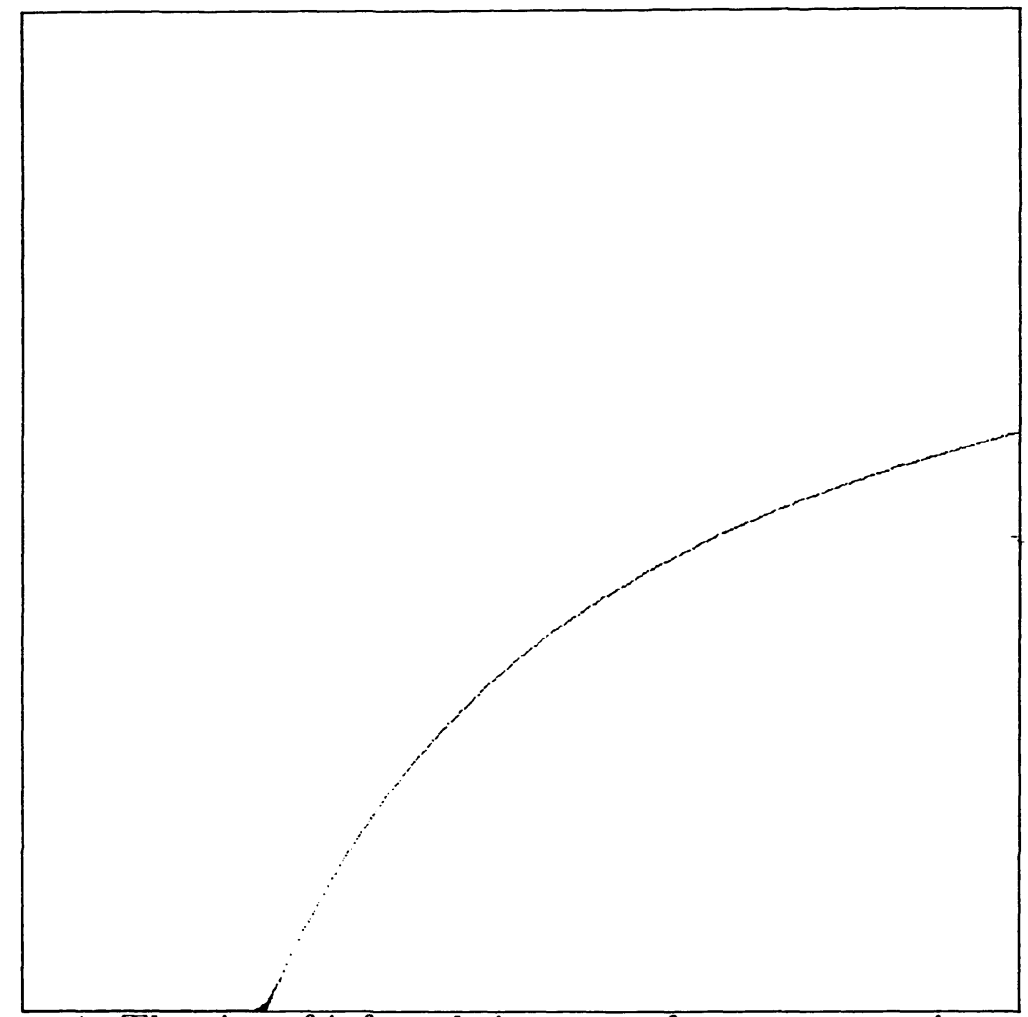

Figure 1: The size of infecteds increases from zero as $\alpha$ increases.

\section{Complex Dynamics In S-I-S Models}

Equations (2) is capable of supporting complex (chaotic) dynamics. For example, if new recruits are governed by Smith-Slatkin model [11, 22],

$$
f(T(n))=\frac{a T(n)}{1+(b T(n))^{l}},
$$

where $a, b$ and $l$ are positive constant.s and $a>1$ then

$$
T(n+1)=\frac{a T(n)}{1+\left(b^{\prime} T(n)\right)^{l}}+\gamma T(n)
$$

where $a$ is maximal per-capita intrinsic growth rate of the population. The parameter $l$ reflects the type and strength of intraspecific competition while 
the parameter $b$ scales the carrying capacity of the population $[13,24]$. Whenever $0<l<\frac{2(a+\gamma)}{a+\gamma-1}$, the total population assumes a positive steady state at $T_{\infty}=\frac{(a+\gamma-1)^{\frac{1}{l}}}{b}$ and Result 2 applies. However, as $l$ increases past $\frac{2(a+\gamma)}{a+\gamma-1}$, the positive equilibrium point undergoes period-doubling bifurcation resulting in a stable 2-cycle (see Figure 2). This process of period doubling bifurcation continues indefinitely.

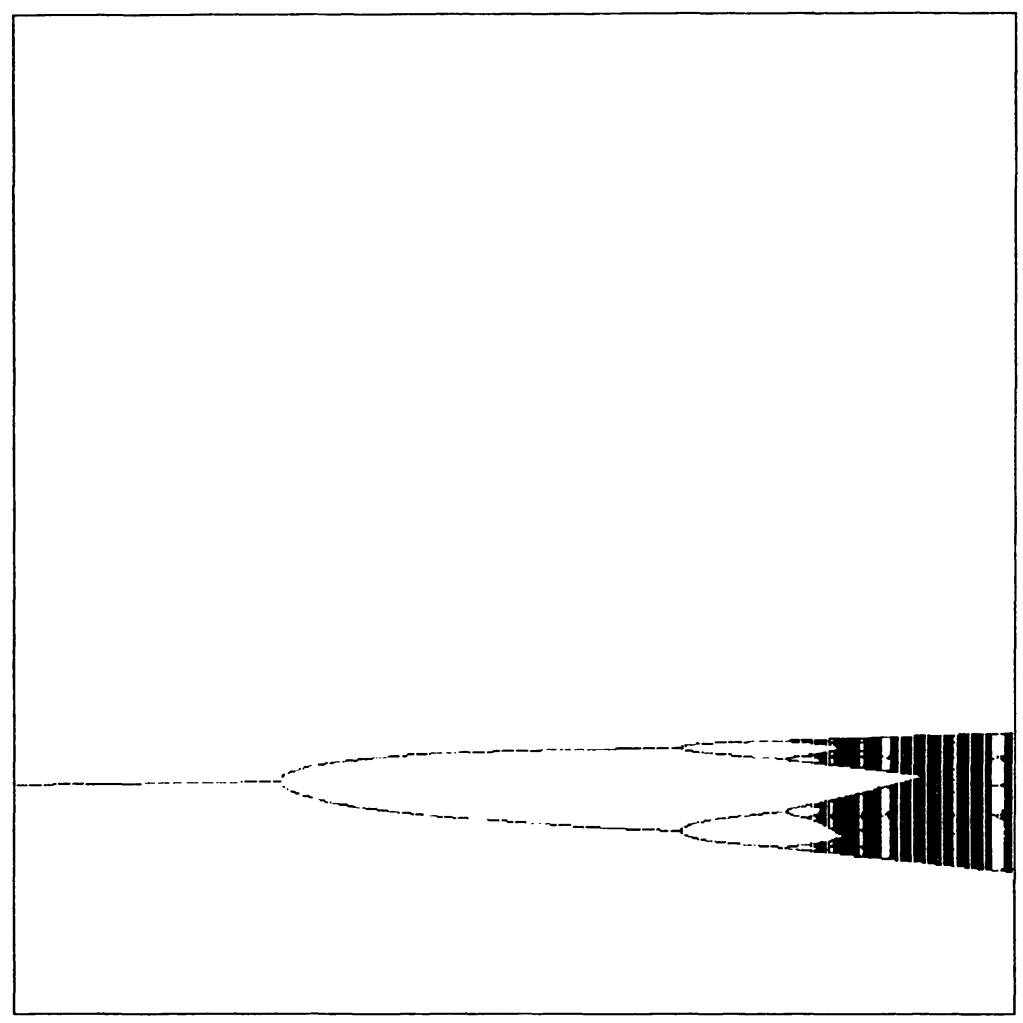

Figure 2: Period-dunbling bifurcations route to chaos in the demographic equation under Smith-Slatkin mudel with $a=1.5, b=1, \gamma=0.1$ and

$$
4.5<l<8.5 \text {. }
$$

Figure 2 illustrates the existence of a chaotic regime in the demographic model. Do complex population dynamics drive disease dynamics? Exam- 
ples in [6] demonstrate that demography does not always drive an epidemic process even in the absence of disease induced mortality.

\section{Backward Bifurcations}

Typically, epidemic mudels have a unique endemic equilibrium with the reproductive number of the disease serving as a threshold parameter (transcritical bifurcation). If the reproduction number is less than one, the disease dies out while if the reproduction number is bigger than one, the disease persists (a forward transcritical bifurcation). If the transmission rate $\alpha_{i} \equiv \alpha_{i}\left(\frac{I_{i}}{T_{i}}\right)$ is non-constant but rather a function of the prevalence then multiple endemic equilibria for System (1) are possible even when the basic reproductive number is less than one (backward bifurcation).

Examples of epidemic models exhibiting this behavior were first established for continuous-time epidemic models by Castillo-Chavez et. al. [7, 8, 14] and Huang et al. [19]. Recently, examples using simpler continuoustime models were constructed by Castillo-Chavez and Hadeler [17], Feng et al. [15], Kribs et. al. [20] and P. van den Driessche et al.[26]. The results of Feng et. al. [15], Huang et. al. [19] and Castillo-Chavez and Hadeler [17] have far reaching implications for the implementation of effective public health policies for HIV and Tuberculusis. The results of P. van den Driessche and P. Watmough have important theoretical implications as they illustrate the possibility of backward bifurcations for simple epidemic processes (S-I$\mathrm{S}$ epidemic models based on a Volterra integral equation [26]). Backward bifurcations are pussible in classical discrete-time epidemic models, that is, when $\alpha$ is constant. An example using a classical (constant $\alpha$ ) discrete-time 
susceptible-exposed-infective-susceptible (S-E-I-S) epidemic model has been recently developed [4].

To illustrate these results $\alpha(y)=y$, that is, $G(\alpha(y) y)=e^{-y^{2}}$. To make the example transparent, we use the approximation $e^{-y^{2}} \approx 1-y^{2}$. Equation (6) becomes

$$
y(t+1)=\frac{\gamma}{\mu+\gamma}(1-y(t)) y^{2}(t)+\frac{\gamma}{\mu+\gamma} \sigma y(t) .
$$

Since $\alpha(y)=y$ is a non-constant transmission rate, prior results do not apply. Linearization of Equation (13) near the disease-free equilibrium $\left(x_{\infty}, y_{\infty}\right) \equiv(1,0)$ gives

$$
y(t+1) \approx \frac{\gamma}{\mu+\gamma}\left(-\alpha(0) G^{\prime}(0)+\sigma\right) y(t) .
$$

Hence, $\Re_{0}=\frac{\gamma \sigma}{\left(1-\Re_{d}\right) \gamma+\Re_{d}}$ where $\Re_{d}=\frac{\mu}{1-\gamma}$. A backward bifurcation using Equation (13) is illustrated numerically. We vary $\Re_{0}$ while the survival probability $\gamma$ and the variable coefficient $\mu$ remain fixed at $\gamma=0.98$ and $\mu=0.01$. The following bifurcation curve (Figure 3 ) is generated: 


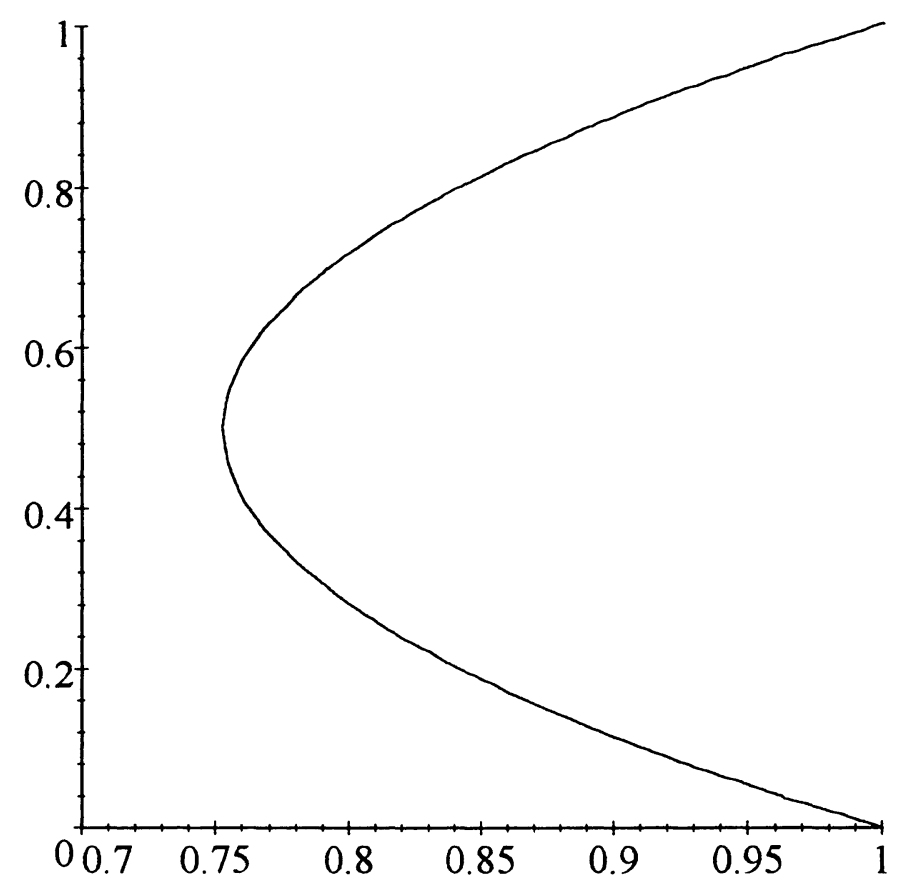

Figure 3: The relationship between the proportion of susceptibles and the basic reproductive number $\Re_{0}$. The horizontal axis, $0.7 \leq \Re_{0} \leq 1$, and the vertical axis, $0 \leq y_{\infty} \leq 1$.

The disease free equilibrium $(1,0)$ is locally asymptotically stable for all values of the parameters. It is globally stable whenever $\Re_{0}<0.7525$. A backward bifurcation urcurs at $\Re_{0} \approx 0.7525$. When $\Re_{0} \approx 0.7525$, an unstable endemic equilibrium appears, and for values of $\Re_{0}$ in the interval $(0.7525,1)$ the system has 2 endemic equilibria coexisting with the disease free equilibrium (see Figure 3). hence, the future of the disease depends on initial conditions, that is, the model supports multiple attractors. 


\section{Conclusions}

Simple nonlinear discrete - time population models are capable of generating complex (chautic) dynamics. Nunlinear discrete-time $(S-I-S)$ epidemic models built on bounded or geometrically growing populations do not generate complex (chautic) dynamics but could generate multiple attractors. We derived thresholds for the persistence of the disease and analyzed the global and local behavior of solutions. Extensions to populations with complex population dynamics and multiple attractors were briefly discussed.

\section{ACKNOWLEDGMENTS}

This study was partially supported by grants from National Science Foundation (NSF Grant DMS 9977919) and National Security Agency (NSA Grant MDA 9149710074). Abdul-Aziz Yakubu was supported in part by the NSF IGERT Program in Nonlinear Systems at Cornell University. This research was completed while Abdul-Aziz Yakubu was a Visiting Scientist at the Center for Applied Mathematics in Cornell University. We thank the office of the provost of Cornell University for its support and Intel Technology for Education 200 Equipment Grant. The examples that lead to this research were part of a summer research experience supported by the above grants. The related work of Barrera, J. H., Cintron-Arias, A., Davidenko, N., Denogean, L. R., Francu-Gonzalez, S. R. and Velazquez, J. P. (under uur supervision) has been published as Biometrics Department Technical Reports (see references). 


\section{Reference}

1. Allen, L. and Burgin, A. M. Comparison of deterministic and stochastic SIS and SIR models, Dept. Math. ES Statistics, Technical Report Series (Texas T'ech. University), (1999).

2. Allen, L. Some discrete-time SI, SIR and SIS epidemic models. Math. Biosci. 124: 83-105 (1994).

3. Anderson, R. M. and May, R. M. Infectious diseases of humans: $D y$ namics and control. Oxford University Press, Oxford (1992).

4. Arreola, R., Crussa, A., Velasco, M. C. Discrete-time S-E-I-S models with dispersal between two patches, Biometric Department, MTBI Cornell University Technical Report. (2000).

5. Bailey, N. T. J. The simple stochastic epidemic: a complete solution in terms of known functions. Biometrika 50, 235-240 (1963).

6. Barrera, J. H., Cintron-Arias, A., Davidenko, N., Denogean, L. R., Franco-Gonzalez, S. R. Dynamics of a two-dimensional discrete-time SIS model, MTHI Cornell University Technical Report (1999).

7. Castillo-Chavez, C., Cooke, K., Huang, W., Levin, S. A. The role of long periods of infectiousness in the dynamics of acquired immunodeficiency syndrome (AIDS), J. Math. Biol., 27, 373-398 (1988).

8. Castillo-Chavez, C., Hethcote, H. W., Andreasen, V., Levin, S. A., Liu, W. M. Cruss immunity in the dynamics of homogeneous and hetero- 
geneous populations. Malhematical Ecology, (ed. L. J. Cross, T. G. Hallam, and S. A. Levin), 303-316 (1988).

9. Castillo-Chavez, C., Yakubu, A. A. Discrete-time $S-I-S$ models with complex dynamics, (Preprint).

10. Castillo-Chavez, C., Yakubu, A. A. Dispersal, Disease and Life-History Evolution, (Preprint).

11. Cooke, K. L., Yorke, J. A. Some equations modelling growth processes and gonurrhea epidemics, Math. Biosci., 16, 75-101 (1973).

12. Cull, P. Local and glubal stability for population models. Biol. Cybern. 54: 141-149 (1986).

13. Doebeli, M., Ruxtun, G. D. Evolution of dispersal rates in metapopulation models: Branching and cyclic dynamics in phenotype space. Evolution, 51(6): 1730-1741 (1997).

14. Dushoff, J., Huang, W., Castillu-Chavez, C., Backwards bifurcations and catastrophe in simple mudels of fatal diseases. J. Math. Bio., 36, 227-248 (1998).

15. Feng, Z., Castillu-Chavez, C., Capurro, A. A model for Tuberculosis with Exugenuus Reinfection, Theor. Popln. Biol. 57, 235-247 (2000).

16. Gonzalez, P. A., Saenz, R., Sanchez, B. The influence of dispersal between two patches on the dynamics of a disease. Biometric Department, M'TBI Cormell Uninersily Technical Report (2000). 
17. Hadeler, K. P., Castillo-Chavez, C. A core group model for disease transmission. Malh. Biosci., 128, 41-55 (1995).

18. Hassell, M. P., Andersun R. C., Cohen, J. E., et al. Impact of diseases on host populations (group repurt). In population biology of infectious diseases, (ed. R. M. Anderson and R. M. May), Springer-Verlag, New York (1982).

19. Huang, W., Couke, K. L., Castillo-Chavez, C. Stabilitiy and bifurcation for a multiple-group model for the dynamics of HIV/AIDS transmission. SIAM J. Appl. Math., 52(3):835-854 (1992).

20. Kribs-Zaleta, C. M., Velasco-Hernández, J. X. A simple vaccination model with a backward bifurcation, Math. Biosci., 164 (2): 183-201 (2000).

21. May, R. M. Simple mathematical models with very complicated dynamics, Nature, 261, 459-469 (1977).

22. May, R. M. Stability and complexity in model ecosystems, Princeton University Press (1974).

23. Nåsell, I. The quasi-stationary distribution of the closed endemic SIS model. Adv. Appl. Prob. 28: 895-932 (1996).

24. Smith, M., Slatkin, M. The stability of predator-prey systems. Ecology, 54:384-391 (1973).

25. Thieme, H. R. Convergence results and a Poincare'-Bendixson trichotomy for asymptotically autonomous differential equations, $J$. Math. 
Biol., 30: 755-763 (1992).

26. van den Driessche, P., Watmuligh, J. A simple SIS model with a backward bifurcation, J. Math. Biol. 40, 525-540 (2000).

27. Velazquez, J. P. SIS nonlinear discrete-time models with two competing strains, MTBI Cornell Universily Technical Report (1999).

28. Zhao, X.-Q. Asymptotic behavior for asymptotically periodic semiflows with applications. Comm. Appl. Nonl. Anal. 3, 43-66 (1996). 